\title{
High Time to Observe on Physical and Emotional Wellbeing of Elderlies: A Narrative Review
}

\author{
Lovesampuranjot Kaur ${ }^{1}$, Maharaj Singh' ${ }^{2}$, Ramandeep Kaur Dhillon ${ }^{3}$ \\ ${ }^{I}$ Ph.D. (Nursing) Scholar, Nims University Rajasthan, Jaipur, ${ }^{2}$ Professor \& Research Head, Department of \\ Nursing, Nims University Rajasthan, Jaipur, ${ }^{3}$ Professor \& Principal, Ajit Nursing Institute, Sunam, Punjab
}

\begin{abstract}
Ageing is a natural process with invitation of multiple challenges and limitations in terms of physical capacity, psychological to social performance of a person. It was estimated that India will be at top of the world in terms of aged population from 2030 to 2050 . This review aimed to assess the overall physical and emotional well-being of elderly population. This was a narrative review, here we have gathered information in a very systematic way. The electronic data base such as ProQuest, Embase, PubMed, PsycARTICLES, EBSCO, ResearchGate, EORTC, Scopus, Educational Resources Information Centre (ERIC), and Allied Health Literature (CINHAL) were searched and article published in between from 2010 to 2020 were identified. A total 978 articles were received from these search engines and based on systematic scrutinizing process a total of 13 articles were included in the review. Reviewed studies findings suggested that elderly stayed at old age home or away from home are facing high challenged to maintain physical and emotional well-being. Then the elderly facing any medical history also found such vulnerable then compare with others. The study concluded with that there is huge need to develop some evidence based intervention which will allow the elderly to meet the minimum need to maintain physical and emotional well-being.
\end{abstract}

Key words: Elderly, physical well-being, emotional well-being, elderly well-being

\section{Introduction}

Aging is a natural process of life which always welcomed with compromised health especially physical and emotional domain of health. One of fact which cannot be neglected that aging having risen everywhere throughout the world and major contributing element is all advances in prescriptions and nursing care and this is of worry to each country to have the option to take care of its old populace. ${ }^{(1)}$

Despite being delicate and helpless, the old could contribute monstrously to the development of the network given that they are appropriately thought about. It's a test for each to instruct them for a sound and

\section{Corresponding Author:}

\section{Ms. Lovesampuranjot Kaur}

Ph.D. (Nursing) Scholar, Nims University Rajasthan, Jaipur, Email- lovedhaliwa191@gmail.com dynamic maturing.

In India, as indicated by Population Census 2011 there are about 104 million older people (matured 60 years or above) in India; 53 million females and 51 million males. This was mentioned that a total of $8.6 \%$ of population shared by elderly in India by year 2016 and estimated to increase in coming years. For an instance, In Punjab it estimated $10.3 \%$ of population share belongs to elderly population. In head counts total of 2866 thousands elder populations live in Punjab in which 1422 thousands are male and remaining 1444 are female elderly. Further 1958 thousands of elderly lives in rural Punjab whereas remaining 908 thousand live in urban Punjab location. Among all states and Union Territory of India, Punjab states falls at $4^{\text {th }}$ numbers in terms of highest old age population share in total population after Kerala, Goa and Tamilnadu. One the reason behind this is to having very good life expectancy at birth and at age of 60 years, as per present data Punjab 
stand at third position after Kerala and Tamilnadu for highest life expectancy at birth which is 69.1 for male and 73.1 for females, whereas at the age of 60 years, for males, Punjab has the highest life expectancy is 19.3 years. $^{(2)}$

In India the aged population is the second largest in the world. The proportion of elderly persons ( $>60$ years) has increased from $4.3 \%$ in 1951 to $7.7 \%$ in 2001 . With current demographic trends it is estimated to reach $21 \%$ by the year $2050 .^{(3)}$

Geriatric care has attracted unprecedented attention and rightly, so as the world is witnessing the phenomenon of global ageing. The most important part of caring for the elderly is to love them and keep them active. There are many different ways to care for the elderly, whether by caring for them in the comfort of their own homes, bringing them to safe environment or moving them to a senior centre. With time and a little hard work, we can properly care for our loved one.

Since the existing literature and statistic about population share of older people among total population of India. One clear indication is drawn out from this literature is that the generation is getting older and number of old people are adding in the list, so it become very important to have enough concentration of wellbeing of this mass population category. As we understand that elderly is always considered under vulnerable population because of many limitations in terms of health. The epidemiological change has brought to the consideration of analysts, experts and strategy creators the significance to advance physical wellbeing and psychosocial prosperity among older residents, whose rate is continuously expanding around the world, even in India at bigger scope. Asset ventures are important to satisfactorily address the requirements of individuals in this life stage, yet additionally to cultivate their strengthening and dynamic commitment in wellbeing advancing practices. ${ }^{(4,5)}$

This review scheduled to understand the physical and emotional wellbeing of elderlies. In broader way, this review will enable the researcher and audience about what kind of challenges and need are facing by elderly population in view to remain their wellbeing.

\section{Materials and Methods}

The literature review was designed as a narrative study, because a broader perception of physical and emotional wellbeing of elderly is different. The articles were included from various countries. A systematic electronic search was used to identify number of studies carried out physical and emotional wellbeing of elderly. The original research papers were only included in study. The following electronic databases are searched: ProQuest, Embase, PubMed, PsycARTICLES, EBSCO, ResearchGate, EORTC, Scopus, Educational Resources Information Centre (ERIC), and Allied Health Literature (CINHAL). The existing literatures were very systematically opted to recruit into this narrative review.

\section{Inclusion Criteria}

1. The research paper only which directly belongs to physical and emotional wellbeing of elderly.

2. The paper which is easily accessible online and full text available.

3. The studies which are completed in English language.

4. Articles included from the year 2010 to 2020 .

\section{Exclusion Criteria}

1. The study concern to with any chronic disease specific.

2. Poor quality journal publications.

3. The research study which is published in without ISSN number journals.

4. The research studies which are not available on journal database.

5. The research studies in which only abstract is available.

6. The studies which is published in local language.

\section{Search Strategy}

MeSH terminology used for PubMed and ERIC("Physical and emotional wellbeing" $\{\mathrm{MeSH}$ Terms $\}$ OR ("physical wellbeing" \{All Fields\} AND 
"emotional wellbeing" \{All Fields\}) OR "wellbeing" \{All Fields\}) AND ("quality of life" \{MeSH Terms\} OR "elderly wellbeing” \{All Fields\}) AND ("old age wellbeing" \{MeSH Terms $\}$.

\section{Results and Discussion}

A total 978 articles were received from search engines from that 675 articles were excluded bases on exclusion criteria. So total retrieved articles were 303 among all 74 duplicate articles, 68 No full text available, 58 not relevant and 51 abstracts were excluded. Final retrieved articles were 52; among them 39 full articles were excluded based on inclusion criteria. Finally, 13 articles were included in the review.

There were many researches organized in view to elderly wellbeing, where they included many dimensions of health. Based on this narrative review, the researcher opted best suited article.

Andrzej knapik et al has published their work in line with relationship between physical fitness andhealth selfassessment in elderly. This was an observational study, in which physical fitness was evaluated using the senior fitness test (SFT). Self-esteem of health was assessed by the sf-36 questionnaire. A total of 123 elderlies were recruited whose age falls in between 60 to 86 years. The study findings were suggested that physical fitness and health self-assessment among elderly may be strongly determined by cultural conditions, for example, habits, lifestyle in various regions. ${ }^{(6)}$

Jharna bag et al stated in their investigation of Assessment of subjective well-being status of elderly people in old age homes in Kolkata in relation to their perceived physical health and cognitive functioning. This was a descriptive survey in which 50 elderly aged more than 65 years were recruited. Elderly was selected by total enumeration sampling from two different old age Home in Kolkata. They were assessed using subjective well-being inventory and a self-developed checklist for perceived physical healthProblems. Cognitive function was assessed by using mini mental state examination. Study result expressed that more than half $(52 \%)$ of the elderly people have reported high subjective wellbeing status. Regression analysis showed that perceived physical health problem $(\mathrm{p}<0.001)$ and having Children $(p=0.010)$ were statistically significant predictors of subjective well-being. In order to improve the quality of life of elderly people health workers should give more emphasis on psychosocial aspects of this population. Improved psychosocial aspects can increase perceived physical health hence subjective well-being. ${ }^{(7)}$

Abdonas tamosiunas et al have shared their work of research in document as psychological well-being and mortality: longitudinal findings from Lithuanian middleaged and older adults' study. The study organized in between 2006-2008. Here 7115 individuals aged 45-72 years were surveyed by questionnaire. Psychological well-being was evaluated by a casp-12 questionnaire. Socio-demographic, Lifestyle, biologic factors and depressive symptoms were evaluated. Study findings stated that Psychological well-being is an important predictor of longevity, controlling well-recognized risk factors such as age, education, cardiovascular diseases, social status, marital status, lifestyle and biological factors and depressive symptoms. Positive psychological well-being should be taken into account when screening older people to prevent negative healthOutcomes. ${ }^{(8)}$

Anjali rathaur, and Sunita Mishra shared their work in line with Psychological and emotional wellbeing in non-institutionalized population. This study was used random sampling technique to recruit the study participants. These were 120 elderly (age group 60-85 years) in which 60 institutionalized and 60 noninstitutionalized.The independent variables were gender, working status and marital status, while the dependent variables were psychological well-being emotional well-being. The research instruments were used as the scale RYFF's (1995) scales of psychological well-Being (SPWB) and use the scale Dr. Shruti Narain (1971) emotional Intelligence scale. (EIS). ANOVA test and Karl-person ' $r$ ' method used to check correlation. The findings revealed that is consistent Relationship between psychological and emotional well-being and health in old age. On applying person's coefficient Correlation significant positive relationship was found between psychological and emotional in elderly people. ${ }^{(9)}$

Yuzana et al. stated in their researched work of reviewing the subjective wellbeing of elderly. This was a cross-sectional study organized at nurul saadah 
institution in Terengganu, Malaysia. Questionnaires were obtained from 73 elderly aged $50-89$ years. The questionnaire was having 28 questions. In the questionnaire, the Likert scale is a five-point scale that is used to allow the participants to express how much they; strongly agree, agree, neither agree nor disagree (or neutral), disagree, or strongly disagree with a particular statement. It communicated based on findings that a majority of the participants reported feeling isolated at their homes rather than in the institution. Emotional and social supports are of minimal significance, while spiritual support is of maximum significance to the elderly population's wellbeing. Furthermore, elderly who maintain frequent contact with their relatives or friends are more optimistic than those who do not maintain such contact. Social networks are significantly correlated with the elderly's wellbeing. ${ }^{(10)}$

Jamila bookwala published her document on Confidant availability (in)stability and emotional well-being in older men and women. Participants in two waves of the Wisconsin longitudinal study were assessed on depressive symptoms and the availability of a family member and friend as confidant.There were 4,631 elderly were recruited in the study. Using mixed linear effects models, four groups were compared over time and across gender on depressive symptoms: those with and without a family/ friend confidant at both waves and those who lost and gained a family/friend confidant.The study revealed that those with stable availability of a family or friend confidant consistently scored the lowest on depressive symptoms; the gain of a family or friend confidant corresponded with a decrease in depressive symptoms, with a larger effect seen for the gain of a family confidant; the loss of a family confidant was associated with an increase in depressive symptoms over time; and stable availability of a family or friend confidant was more strongly linked to lower levels of depressive symptoms among women, whereas stable unavailability of a family confidant was linked to higher levels of depressive symptoms. ${ }^{(11)}$

Priyanka thakur et al. stated in their investigated work on quality of life and psychological well-being among elderly living in old age homes and living with their families in selected areas of Uttarakhand. A quantitative research approach with descriptiveComparative design was used to assess the quality of life and psychological well-being among elderly living in old age homes and living with their families. There were total 164 elderly were recruited. The population consisted of old people residing in selectedOld age homes and community area. The study was conducted at selected old age homes and community area of Uttarakhand. Purposive sampling technique was used to recruit the 164 subjects from the population i.e., 82 subjects from respective old age homes and 82 subjects from selected community area. Sociodemographic proforma, who QOL-BREF scale and self -developed psychological well-being scale was used to assess the QOL and psychological well-being among elderly with the help of structured interview method. The result of the study shows that overall mean score of QOL for elderly living in old age homes and those living with their families was 68.47 and 97.43 respectively. Similarly, overall mean score for psychological wellbeing among elderly living in old age homes and those living with their families was 45.31 and 66.86 respectively which showed that QOL and psychological well-being was good among those elderly who were living with their families as compare to old age homes inmates. On comparison of overall mean of QOL it was found that mean score for QOL with SD for elderly living in old age homes and living with families was $68.47 \pm 10.752$ and $97.4 \pm 8.564$ similarly on comparison of overall mean score of psychological well-being it was found that score was $45.32 \pm 6.385$ and $66.87 \pm 5.86$ which was found to be statistically significant at $p \leq 0.05$. Hence the overall findings suggest that quality of life and psychological well-being was good among elderly who were living with their families as compare to those who were living in old age homes. ${ }^{(12)}$

Jinmyoung cho et al has communicated their researched work in line with the relationship between physical health andPsychologicalwell-being among oldest-old adults. There were 306 older people were identified as study participants. Structural equation modelling was performed to examine health influences on psychological well-being. Latent variables were created to reflect subjective health, as measured by self-ratings of health and objective health, as measured by physical health impairment (i.e., health problems, pastAnd 
present diseases, hospitalization) and biomarkers (i.e., haemoglobin and albumin). Psychological well-being was measured by positive and negative affect. The analysis of study expressed that there were significant direct effects of subjective health on affect and significant indirect effects of objective health through subjective health on positive affect and negative affect. Subjective health took the role of a mediatorbetween objective health and psychological well-being. These results highlight the status and perceptions of health as a criticalIndicator for well-being in extreme old age. ${ }^{(13)}$

N.N. Ariati et al. has communicated their work on the ergonomic elderly gym improving physical fitness and increasing the bone mass of the elderly. There were 20 elderly selected as study participants in the study. The data collections were physical fitness and bone mass. The difference in treatment effects was analysed using a paired sample t-test with $\alpha=0.05$ for data with normal distribution and Wilcoxon test, $\alpha=0.05$ for data with the abnormal distribution. Result has showed that there were differences in physical fitness improvement in period $i$ and period ii after 8 weeks of gymnastics at $43.43 \%$ with very bad categories being bad, the difference in the increase in whole-body bone mass was $16.76 \%$ and leg bone mass $68.67 \%$. Analysis of paired sample-t-test physical fitness data and Wilcoxon test for bone mass data in period $\mathrm{i}$ and period ii after gymnastics for 8 weeks, found that there was a significant difference $(\mathrm{p}$ $<0.05)$. It can be concluded that ergonomic elderly gym can significantly improve physical fitness and increase the bone mass of the elderly. It is recommended that the elderly continue to exercise with a duration of $30-45$ minutes, the frequency of three times a week to maintain the elderly's physical fitness. ${ }^{(14)}$

Pipit Festi Wiliyanarti et al shared their researched work on developing holistic care model: the physical wellbeing of elderly based on social support and characteristic. The sample's age was more than sixty years old, living with their family and under Medokan Ayu public health centre supervision. Total 110 samples were selected to perform the study. Multi stage random sampling was performed. The research instrument was physical wellbeing including elderly autonomy, cognitive, complaining about physical and disease as well. Structural equation modelling with partial least square was used to analyse the data.The research results showed that majority of emotional support in social support variable was good $(98 \%)$. The majority of instrumental support was good $(88.18 \%)$. Social wellbeing has significant relationship with the elderly physical wellbeing (p 0.312, t-statistic: 4.420 , t-table: $1.65)$, elderly characteristic of holistic care (0.178, t-statistics 2.422 , t-table: 1.65 ), and elderly characteristic of physical health (0.140. T-statistic 1.790, t table: 1.65$)$. Finally, it concluded that social support influences the physical wellbeing of elderly. ${ }^{(15)}$

Yi-Ju Lee \& Wei-Li hung have communicated their research work on the relationship between exercise participation and well-being of the retired elderly. Here total of 352 retired older were recruited. Face-to-face questionnaire survey was adopted, and quota sampling was chosen to select the respondents. A total of 352 valid questionnaires were collected in selected parks in Taipei.The results showed that exercise frequency and well-being were positively correlated, but a negative correlation was found between exercise intensity and well-being. The survey found that the intensity of exercise was self-evaluated by as being low to moderate, but most of the activities were in the categories of moderate or vigorous intensity according to the metabolic equivalents suggested by American college of sports medicine. The study suggest that the elderly felt more comfortable and gained more pleasure psychologically while participating in exercises less intensive. As a result, the retired elderly are recommended to take exercise as frequently as possible. As to exercise intensity, selfevaluated low-to-moderate intensity exercise might be better for the psychological well-being of the elderly. ${ }^{(16)}$

Zoran Milanović et al has published their work with age-related decrease in physical activity and functional fitness among elderly men and women. In study 1288 elderly were participated. Participants' level of physical activity was evaluated using the International physical activity questionnaire: 594 were male and 694 females. Functional fitness was also estimated using the senior fitness test: back scratch, chair sit and reach, 8-foot up and go, chair stand up for 30 seconds, arm curl, and 2-minute step test.This study found that the reduction in physical 
activity level and functional fitness was equal for both men and women and was due to the aging process. These differences between Young and old elderly people were due to the reduction of muscle strength in both upper and lower limbs and changes in body-fat percentage, flexibility, agility, and endurance. ${ }^{(17)}$

Abdel-Hady el-Gilany and Raefa Refaat Alam shred their investigated work as effects of nursing program as a life review on life satisfaction and happiness among elderly people. It was a quasi-experimental study having study settings as two elderly clubs namely; Elsaada and el-amal in Mansoura, Egypt. Data collected from 74 older people, and it was with socio-demographics, subjective happiness, and satisfaction with life scales. Nursing program as (a life review) was implemented and the twoScales were re-measured immediately and two months after intervention. Findings evidenced that there is an improvement of the overall median life satisfaction score in the immediate post-intervention and two months after intervention. These improvements are statistically significant with $\mathrm{p}=0.001$. Also, the overall median happiness score improved from 14 up to 19.5 and 20 in the immediate and two-months postintervention; respectively. These improvements are statistically significant with $\mathrm{p}=0.001$. This pattern of improvement persists after stratification of all the sociodemographic variables studied and with the presence or absence of chronic diseases. Finally, it concluded that implementation of a life review intervention improved the life satisfaction and sense of happinessamong elderly immediately and post 8 weeks of intervention. ${ }^{(18)}$

\section{Conclusion}

Researcher made all attempts to gather all relevant information in view to assess the present condition of physical and emotional well-being of the elderly. Throughout the review process, researcher came in impression that majority of elderly lives in old age home or such similar institutions are facing multiple health issues from physical illness to psychological distress and social stigma. But it also noticed at elderly either remain in institution or non-institution area but they are facing physical and emotional distress, which is again irrespective of country, as this review covers reviews from not only India but from developed to undeveloped countries. This review made a way for researcher to take up conclusion from this review to develop some intervention which can be helpful to elderly to improve their physical and emotional well-being.

Acknowledgement: Researcher would like to acknowledge untried support and guidance of NIMS university along with $\mathrm{My} \mathrm{PhD}$ Supervisor and cosupervisor.

Ethical Clearance: Taken from Institutional Ethical Committee, National Institute of Medical Science and Research, NIMS University, Jaipur, Rajasthan via Ref. No.: NIMSUNI/IEC/2018/PHD/116.

Source of Funding: Self-funded project

\section{Conflict of Interest: Nil}

\section{References}

1. Chida Y, Steptoe A. Positive psychological wellbeing and mortality: A quantitative review of prospective observational studies. Psychosom Med. 2008;70(7):741-56.

2. Khan S, Itrat M. Current Issues in Geriatric Health Care in India-A Review. J Community Med Heal Care. 2016;1(1):1003.

3. Park K. Essentials of Community Health Nursing. 23rd ed. Jabalpur: M/s Banarsidass Bhanot; 2002. 656-58 p.

4. Delle Fave A, Bassi M, Boccaletti ES, Roncaglione C, Bernardelli G, Mari D. Promoting well-being in old age: The psychological benefits of two training programs of adapted physical activity. Front Psychol. 2018;9(MAY):1-13.

5. Kadariya S, Gautam R, Aro AR. Physical Activity, Mental Health, and Wellbeing among Older Adults in South and Southeast Asia: A Scoping Review. Biomed Res Int. 2019;2019:11.

6. Knapik A, Brzęk A, Famuła-Wąż A, GallertKopyto W, Szydłak D, Marcisz C, et al. The relationship between physical fitness and health self-assessment in elderly. Medicine (Baltimore). 2019;98(25):e15984.

7. Bag J, Chakrabarti A, Daniel L, Sanyal D. Assessment of subjective well-being status of elderly people in old age homes in Kolkata in relation to their perceived physical health and 
cognitive functioning. J Ment Heal Hum Behav. 2014;19(1):32.

8. Tamosiunas A, Sapranaviciute-Zabazlajeva L, Luksiene D, Virviciute D, Peasey A. Psychological well-being and mortality: longitudinal findings from Lithuanian middle-aged and older adults study. Soc Psychiatry Psychiatr Epidemiol. 2019;54(7):80311.

9. Rathaur A, Mishra S, ScholarMP, Studies F, Bhimrao B, Pradesh U, et al. Psychological and emotional well-being in non-institutionalized population. Int J Humanit Soc Sci Res. 2016;2(10):49-54.

10. Yuzana D, Zakirah, Rabiatul. Reviewing the subjective wellbeing of elderly. Int J Adv Res Innov Ideas Educ · [Internet]. 2020;6(3):1060-4. Available from: www.ijariie.com

11. Bookwala J. Confidant Availability (In)Stability and Emotional Well-Being in Older Men and Women. Gerontologist. 2017;57(6):1041-50.

12. Priyanka Thakur $\mid$ Grace M. Singh $\mid$ J. ManoRanjini. Quality of Life and Psychological Well Being among Elderly Living in Old Age Homes and Living with their Families in Selected Areas of Uttarakhand. Int J Trend Sci Res Dev [Internet]. 2019;3(6):1033-8. Available from: https://www.ijtsrd.com/papers/ ijtsrd29146.pdf\%0Ahttps://www.ijtsrd.com/otherscientific-research-area/other/29146/quality-of- life-and-psychological-well-being-among-elderlyliving-in-old-age-homes-and-living-with-theirfamilies-in-selected-areas-of-uttara

13. Cho J, Martin P, Margrett J, MacDonald M, Poon LW. The relationship between physical health and psychological well-being among oldest-old adults. J Aging Res. 2011;2011.

14. Ariati NN, Adiputra N, Tirtayasa K, Adiatmika IPG, Pangkahila A, Sutjana ID., et al. the Ergonomic Elderly Gym Improving Physical Fitness and Increasing the Bone Mass of the Elderly. Int J Ind Eng Eng Manag. 2020;1(2):1.

15. Wiliyanarti PF, Asri A, Putra KWR. Developing Holistic Care Model: the Physical Wellbeing of Elderly Based on Social Support and Characteristic. Public Heal Indones. 2018;4(3):108-15.

16. Yi-Ju Lee, Hung W-L. The relationship between exercise participation and well-being of the retired elderly. Aging Ment Health. 2011;15(7):2011.

17. Milanović Z, Pantelić S, Trajković N, Sporiš G, Kostić R, James N. Age-related decrease in physical activity and functional fitness among elderly men and women. Clin Interv Aging. 2013;8:549-56.

18. El-Gilany A-H, Alam RR. Effects of Nursing Program As A life Review on Life Satisfaction And Happiness Among Elderly People. IOSR J Nurs Heal Sci. 2017;06(03):15-21. 